\title{
The lazy shadow: a monocular counterpart to the Pulfrich stereo phenomenon
}

\section{Lars Frisén}

Background: The Pulfrich phenomenon is a dynamic stereo dysmetropsia attributed to an asymmetry of neural conduction between the eyes. The phenomenon may arise spontaneously with ocular and neurological disease and may be induced in normal subjects by placing a light-attenuating filter before one eye. By analogy, it is predicted that a localised variation of retinal illumination within one and the same eye should affect the perception of moving targets.

Methods: A rotating, nesting square display was generated by computer graphics. The inner square was painted bright white, the outer dim grey. Luminances, rates of rotation and angular sizes were varied.

Results: On rotation, the outer, dimmer square appeared to lag behind the inner, brighter one, as a "lazy shadow". The lag was measured quantitatively in normal observers by applying a compensatory lead to the lagging square. The magnitude of lag was found to depend on luminance, spin rate and visual angle. Lags exceeding $10^{\circ}$ were observed under optimum conditions. Conclusions: The experimental results confirm the existence of a monocular counterpart to the binocular Pulfrich phenomenon. Distortions of moving images are likely to occur spontaneously with monocular, localised visual field defects.

$T$ he Pulfrich phenomenon is a distortion of dynamic stereo vision that may occur in a number of ocular and neurological disorders. The phenomenon also can be induced in normal subjects by placing a light-attenuating filter before one eye. The common denominator to these seemingly disparate settings is thought to be an asymmetry of rates of neural conduction between the eyes, resulting in a skewed stereoscopic disparity. The perceptual corollaries are apparent distortions of paths of moving objects. Such distortions may have important consequences in daily life, for example, in sports and in traffic. Their magnitudes depend mainly on the degree of interocular asymmetry and the velocity of the moving object. For reviews, see Diaper, and Howard and Rogers. ${ }^{12}$

It is pertinent to ask whether perceptual delays might occur also monocularly, with circumscribed visual field defects. A localised retardation of neural conduction might arise spontaneously with localised disease. A localised reduction of retinal illumination should have the same effect. If so, movement of the retinal image would be expected to generate an apparent localised lag, or a localised distortion of shape, or both.

Unlike the binocular Pulfrich phenomenon, a monocular counterpart cannot easily be induced by means of filters. A better way to manipulate local retinal illumination is to manipulate local luminances of test targets in computer graphics. There are many possible arrangements. One is to present a pair of nesting squares. The inner, smaller square is painted bright white whereas the outer, larger square is painted dim grey. While stationary, the two squares have the expected appearance but when applying rotation around the common centre, the outer dimmer square appears to lag behind, in the form of a "lazy shadow". The perceived lag can be measured quantitatively by applying a compensatory lead angle to the dim square. The lazy shadow phenomenon is quite striking and may have important implications for understanding abnormal vision. This report presents a first exploration of the key parameters, luminance, spin rate and size, in normal observers.

\section{SUBJECTS AND METHODS}

\section{Subjects}

Subjects were recruited from staff. A history of ocular or neurological disease or an abnormal finding in a neuroophthalmological examination were grounds for exclusion. Detailed measurements were obtained from two subjects (A and B), aged 46 and 67 , and spot checks from a third subject, aged 52. Qualitative observations were obtained from an additional four subjects (aged 34-64). All provided informed consent. Ethics committee approval was not required.

\section{Methods}

Computer graphics displays consisting of rotating, solid, nesting shapes with different luminances (fig 1) were presented on a SyncMaster 700b display (Samsung, Upplands Väsby, Sweden), in an otherwise dark room. Details of test conditions will be presented in the Results section. All tests began with the presentation of a set of targets where a randomly selected lag or lead angle was applied to the dimmer rim target. The test task was to adjust the angle so that the core and rim borders appeared to be exactly parallel, by clicking on the computer mouse. The step factor was $1^{\circ}$. Viewing time was unlimited. There was no specific fixation point. The non-tested eye was covered with an opaque patch. The direction of rotation was changed at random between tests. Luminances were measured with an S-1 Universal Photometer (B Hagner AB, Solna, Sweden).

The software was programmed in Java and placed on the departmental internet server (http://www.oft.gu.se/webdiagnos), termed "the website" in the following text. Visitors should note that there are considerable photometric variations between different computer display units and their performance cannot be sensed remotely. Users desiring reproducible measurements need to calibrate their displays.

\section{RESULTS}

The software programming of rotating targets is straightforward but the generation of rotating images on a computer display highlights several technical shortcomings. Most types of targets are subjected to complicated distortions and ghosting, particularly at rotation rates exceeding some 30 revolutions per minute (RPM). Generally, old-fashioned cathode ray tubes (CRT) appear to perform better than liquid crystal displays

Abbreviations: CRT, cathode ray tubes; LCD, liquid crystal displays; RPM, revolutions per minute 

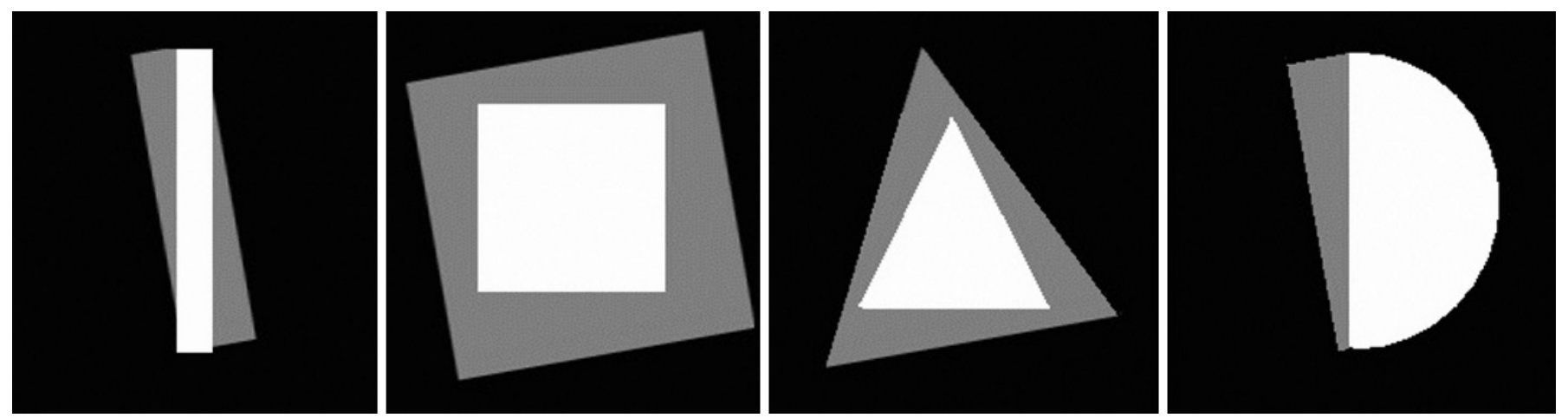

Figure 1 Drawing of different targets as they might appear on clockwise rotation: the dimmer rims lag behind the brighter cores. Lag angle $=10^{\circ}$.

(LCD) in this regard. All results presented here were obtained with a CRT.

Initial explorations used a bar target (fig l, left), which rendered well at low rates of rotation. However, a distracting, swirling pattern appeared at higher rates and motivated switching to a more robust, square target (fig 1, second from left). All measurements reported here were made using this configuration. Subjectively, the triangular target was more difficult to judge, but on the whole all three types produced comparable results. After measurements were completed, a fourth target configuration was discovered (fig l, right), which appeared to be particularly resistant to display glitches.

The desired localised reduction of retinal illumination was achieved by setting the luminance of the target rim to a level lower than that of the target core. The latter was kept at near maximum of display output, $140 \mathrm{~cd} / \mathrm{m}^{2}$. Not unexpectedly, different rim settings produced different apparent lags. At 39 RPM, the maximum lag occurred at the lowest rim setting, $1 \mathrm{~cd} / \mathrm{m}^{2}$, and the lag tapered gradually towards zero near $70 \mathrm{~cd} /$ $\mathrm{m}^{2}$ (fig 2). Variation appeared independent of luminance level.

The lag magnitude varied not only with luminance but also with the rate of rotation (fig 3). Varying the viewing distance revealed that visual angle also played an important role, with a gradual decrease in lag angle with decreasing angular subtense (fig 4). Complete sets of measurements could not be obtained at

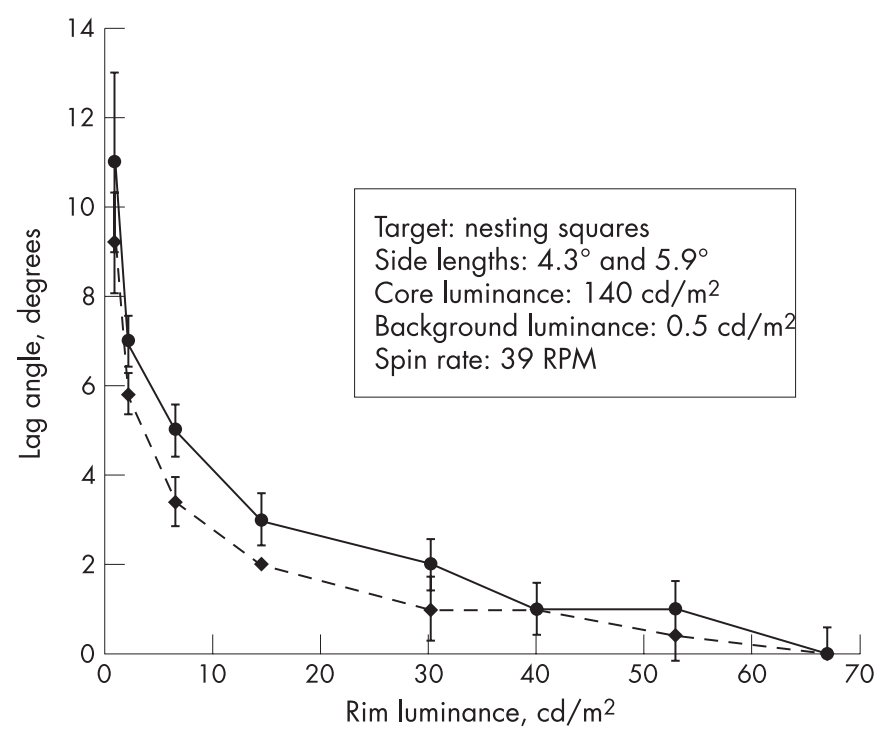

Figure 2 Observed lag angles versus luminance. Means of five measurements from subjects A (broken line) and B (solid line). Vertical bars represent standard deviations. Inset shows test parameters. angles smaller than $1^{\circ}$ because target rims became invisible at the lowest luminance settings.

Using mid-range parameter settings, all participating subjects could easily see the lazy shadow phenomenon. The three subjects who contributed detailed measurements showed some systematic differences as to magnitudes of lag (figs 2-4).

In summary, the lazy shadow phenomenon was markedly influenced by several target variables, namely, luminance, rate of movement and visual angle. The phenomenon was most striking with large contrast differences, rotation rates $\geqslant 30$ RPM, and visual angles $\geqslant 6^{\circ}$. For dynamic demonstrations of the phenomenon, visit the website (see Methods section for details).

One variable remained uncharted in this study, namely, the background luminance. Informal explorations revealed that lag phenomena may also occur on bright backgrounds. The interaction of all luminance variables (background, core and rim) merits further study.

\section{DISCUSSION}

The classical Pulfrich phenomenon has attracted considerable attention but the possibility of a monocular counterpart appears to have been addressed by one study only. ${ }^{12}$ Feinsod and colleagues aimed to induce a localised neural transmission delay in normal subjects by placing a light-attenuating filter so that it covered only one-half of the pupil. ${ }^{3}$ Viewing the classical Pulfrich stimulus, a pendulum swinging in the frontal plane, their subjects reported that the filter added depth to one-half of the pendulum path, with a trajectory somewhat like a

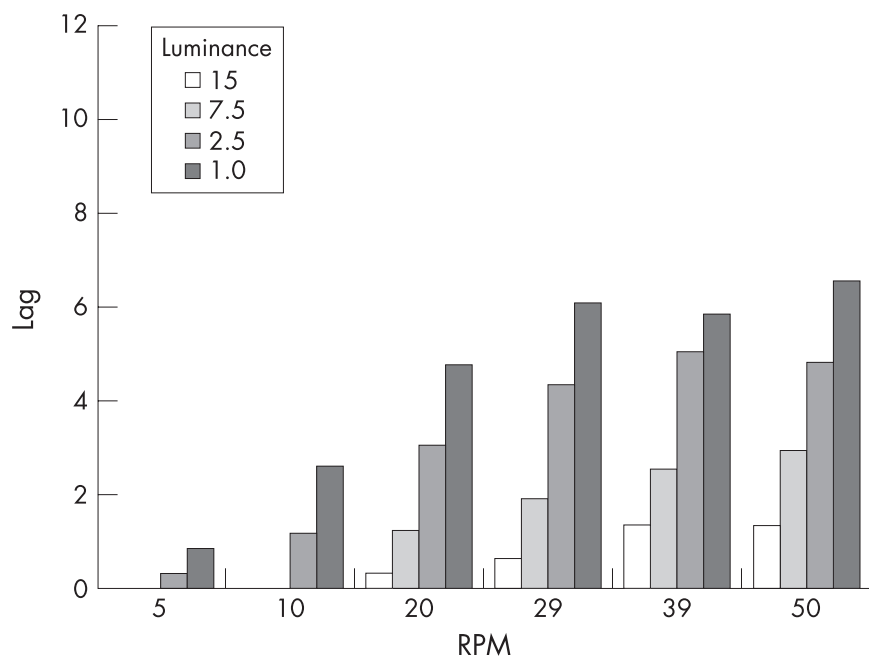

Figure 3 Observed lag angles versus rates of rotation for subject A. Rim subtense $5.9^{\circ}$. Means of three measurements. RPM, revolutions per minute. 


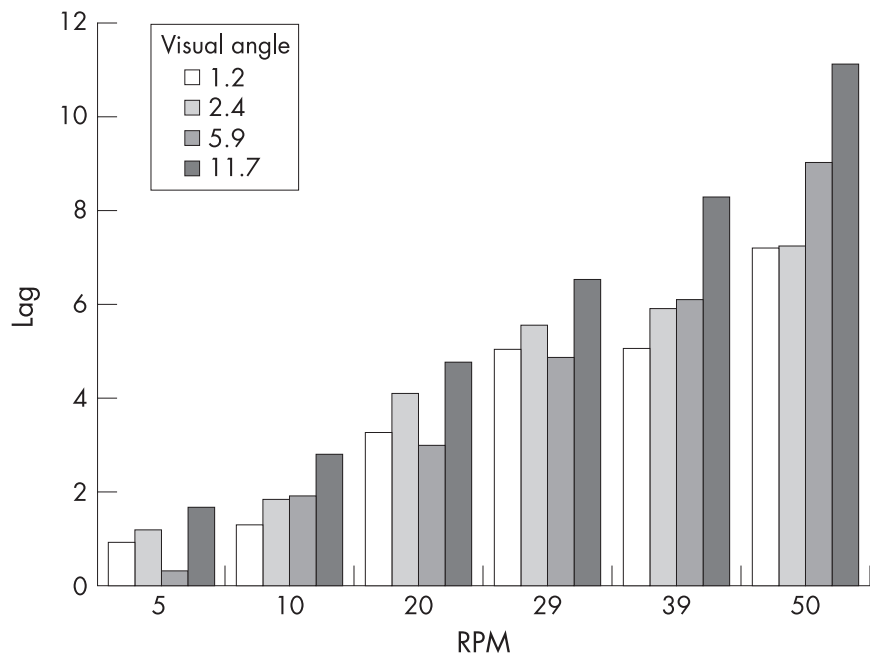

Figure 4 Observed lag angles versus rates of rotation for subject $B$. Core angle was $75 \%$ of the rim angle. Rim luminance $2.5 \mathrm{~cd} / \mathrm{m}^{2}$. Means of three measurements. RPM, revolutions per minute.

recumbent figure nine. Because stereoscopic disparity cannot be generated in a monocular setting, this is an unexpected result. Independent confirmation is lacking.

Prediction of the appearance of a pendulum swinging in and out of a localised visual field defect is a complicated matter and is best approached piecemeal: what might happen on traversal of the defect border and what might happen inside the defect? It seems reasonable to expect that the pendulum should momentarily seem to decelerate and then accelerate on entrance into the defect and vice versa on exit. However, the magnitudes of these "stutters" are likely to be vanishingly small and also counteracted by perceptual completion phenomena. While inside the defect borders, the pendulum's apparent rate of movement should be unaffected, unless there is considerable local variation of defect depth. On these grounds, a pendulum-based test would appear to have little potential to uncover dynamic corollaries of monocular field defects.

Local manipulation of retinal illumination is a more straightforward approach and has been employed previously in laboratory settings, usually using linear target movements and brief exposure times. Typically, dim targets appear to move at lower velocities than their bright counterparts (but the opposite may occur under certain conditions). ${ }^{4}$ It may be tempting to interpret the lazy shadow phenomenon in a similar way. However, the target's component parts (core and rim) do not appear to revolve at different rates: the rates are the same but the one lags behind the other, with a constant lag angle.

Variations on the rotation approach introduced here should be more informative than pendulum-based tests, not least because of the possibility of nulling. Consider an eye with a right hemifield depression that fixates on the centre of a straight bar. Further, let the bar rotate around the fixation point. Most of the time, one half of the bar will be seen to move in the normal hemifield and the other half in the abnormal hemifield. If the latter suffers a retarded neural transmission, this part of the bar should appear to lag behind the other part, as if the whole bar was slightly bent in the middle. Further, the whole bar should appear to stutter slightly when crossing the vertical meridian. These effects should be nullable by applying an adjustable lead angle within the depressed hemifield. A dynamic demonstration of these ideas is available at the website where also a practicable test display can be found.

Prediction of the nature of acquired lazy shadows seems fairly straightforward for localised field defects with simple shapes and uniform depths. However, defects with complicated shapes and non-uniform depths, for example, arcuate scotomata, are presumably associated with quite complicated varieties of lazy shadows. Furthermore, field defects with complicated shapes are often bilateral and also asymmetric, suggesting exceedingly complicated subjective corollaries.

An acquired binocular Pulfrich phenomenon may be nulled by placing an appropriate light-attenuating filter before the quicker eye. ${ }^{5}$ Unfortunately, there seems to be no comparable remedy for monocular lazy shadows.

Competing interests: None.

Correspondence to: Lars Frisén, Institute of Neuroscience and Physiology, Section of Neurological Sciences and Rehabilitation, The Sahlgrenska Academy, Göteborg University, Sweden; lars.frisen@neuro.gu.se

Accepted 3 April 2007

Published Online First 12 April 2007

\section{REFERENCES}

1 Diaper CJ. Pulfrich revisited. Surv Ophthalmol 1997;41:493-9.

2 Howard IP, Rogers BJ. Binocular vision and stereopsis. Oxford: Oxford University Press, 1995:535-54.

3 Feinsod M, Bentin S, Hoyt WF. Pseudostereoscopic illusion caused by interhemispheric temporal disparity: clinical and experimental observations. Arch Neurol 1979:36:666-8.

4 Thompson P, Brooks K, Hammett ST. Speed can go up as well as down at low contrast: implications for models of motion perception. Vision Res 2006;46:782-6.

5 Heron G, Thompson KJ, Dutton GN. The symptomatic Pulfrich phenomenon can be successfully managed with a coloured lens in front of the good eye: a longterm follow-up study. Eye 2006 June 9 [Epub ahead of print]. 